\title{
The Conformance Analysis on Bending Forming of Honeycomb Carton
}

\author{
Qiong Chen* and Yuanjun Lv
}

Zhejiang Industry Polytechnic College, No.151 Qu-Tun load, Shaoxing, 310012, China

\begin{abstract}
Geometry constructions of honeycomb core are finding increasing use because of their relative advantages over other structural materials in terms of improved stability, high stiffness to weight and strength to weight ratios. It provides an efficient solution to increase bending stiffness without significant increase in structural weight. However, honeycomb carton, as a new product of paper honeycomb, has been made by bending forming and failure process of paper honeycomb. Theoretical analysis on bending mechanical properties of paper honeycomb has been done. Then a model has been built on finite element analysis (FEA) software and analyzed its nonlinear bending process. The shear deformation process of honeycomb carton may be approximately categorized into four phases: linear elastic phase, elastic-plasticity phase, plasticity collapse phase, densification and fracture phase. In order to get precise results, not only shear force but also the failure process of paper-core should be considered. Therefore, the curve of external force and the shear deformation process has been analyzed. In addition, stress concentrations due to bending load and cohesive force have been eliminated by drying process of honeycomb carton. At last, the results are introduced by experiment and put into practice.
\end{abstract}

Keywords: Bending behavior, drying process, failure mechanism, honeycomb core, shear process.

\section{INTRODUCTION}

Paper honeycomb is one kind of new branch in packing industry. Due to their efficient structural geometry and energy absorption characteristics, paper honeycomb is widely used in aviation products, packaging, military affairs, construction, vehicle and so on. The main function of honeycomb core is not only to maintain the distance between the upper face-sheet and the lower one, and strengthen the bending stiffness of sandwich, but also carries shear stress in both the longitudinal and transversal directions.

By analyzing existing researches, it was found that the performance study of paper honeycomb was mainly focused in the performance index, static and dynamic characteristics, theoretical modeling and finite element modeling analysis, etc. [1-13]. For example, Dynamic and static compression tests for paper honeycomb cores and absorbed energy were gotten by Kobayashi H [4]. Wang Dongmei [5] deduced that the cushioning properties of paper honeycombs were mainly decided by the value of plateau stress and the length of plateau strain section during compression process. The effect of relative humidity on the energy absorption characteristics of honeycomb paperboard was investigated experimentally by E Yuping and Wang Zhiwei [6]. A mathematical model was developed to describe the relationship between the energy absorption properties of paper honeycombs and ambient humidity, as well as the structural parameters thereof by ZhiWei Wang and Yu Ping E [7]. Gibson [8] deduced the honeycomb material equivalent parameter, the relative elasticity modulus, the shear modulus and Poisson's ratio. Fu Minghui

\section{THEORY}

\subsection{Mechanical Properties}

Honeycomb core supports two face-sheets and is in possession of rigidity, compressive resistance and shearing resistance. The paper honeycomb can be grouped as a cellular material. When one calculates the cellular material as being generally equivalent to the continuous medium, the merit is that there is a minimum amount of calculation involved, but the precision of the calculation is insufficient. 


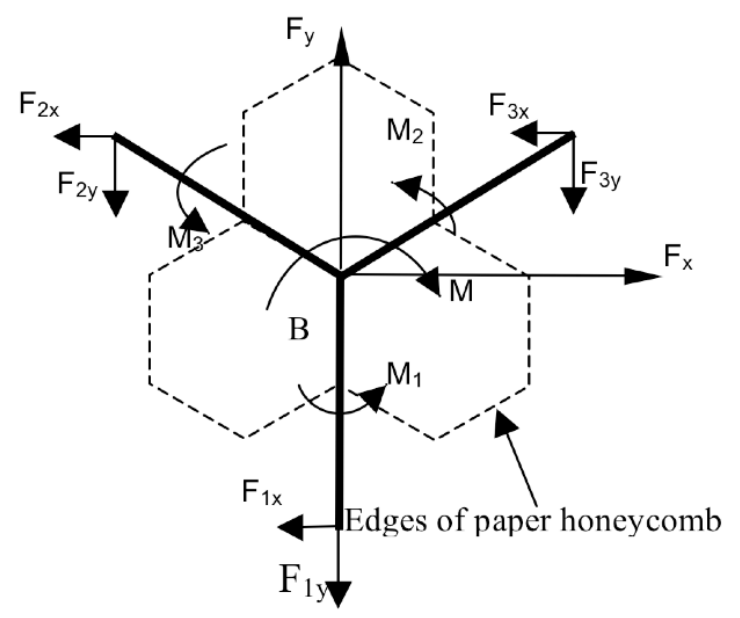

Fig. (1). A continual honeycomb grid model.

The honeycomb structure can be deconstructed by using a continual honeycomb grid model consisting of a "Y" node shown in Fig. (1). Using this coordinate system, the displacement of node A is selected from the node (i,j) to serve as a basic parameter. $u$ and $v$ are defined to be positive bending when they are measured the forward direction from left to right, and $\varphi$ is defined to be positive when it is measured in the clockwise direction. The governing equation of the honeycomb structure "Y" node can be obtained by using its balance equation. The governing equations are as follows:

$$
\begin{aligned}
& F_{x}=\frac{6 E I}{l_{y}^{2}}[\varphi(i, j)+\varphi(i, j-1)]-\frac{12 E I}{l_{y}^{2}} u_{\bar{y}}- \\
& \left(E A \cos ^{2} \theta+\frac{12 E I}{l^{2}} \sin ^{2} \theta\right)\left(u_{\bar{\theta}}-u_{-\bar{\theta}}\right) \\
& -\left(E A-\frac{12 E I}{l^{2}}\right) \sin \theta \cos \theta\left(v_{\bar{\theta}}+v_{-\bar{\theta}}\right)+ \\
& \frac{6 E I}{l^{2}} \sin \theta[\varphi(i-1, j)+2 \varphi(i, j)+\varphi(i+1, j)] \\
& F_{y}=E A u_{\bar{y}}-\left(E A-\frac{12 E I}{l^{2}}\right) \sin \theta \cos \theta\left(u_{\bar{\theta}}+u_{-\bar{\theta}}\right)+ \\
& \left(E A \sin { }^{2} \theta+\frac{12 E I}{l^{2}} \cos ^{2} \theta\right)\left(-v_{\bar{\theta}}+v_{-\bar{\theta}}\right) \\
& +\frac{6 E I}{l^{2}} \cos \theta[\varphi(i-1, j)-\varphi(i+1, j)] \\
& M=4 E I\left(\frac{1}{l_{y}}+\frac{2}{l}\right) \varphi(i, j)+2 E I\left(\frac{\varphi(i, j-1)}{l_{y}}+\frac{\varphi(i+1, j)}{l}+\right. \\
& \left.\frac{\varphi(i-1, j)}{l}\right)-\frac{6 E I}{l_{y}} u_{\bar{y}}+\frac{6 E I}{l} \sin \theta\left(-u_{\bar{\theta}}+u_{-\bar{\theta}}\right)+ \\
& \frac{6 E I}{l} \cos \theta\left(v_{\bar{\theta}}+v_{-\bar{\theta}}\right)
\end{aligned}
$$

where, $\quad u_{-\bar{\theta}}=[u(i, j)-u(i-1, j)] / l, \quad u_{\bar{\theta}}=(u(i+1, j)-$ $u(i, j)) / l ; \theta$ is included angle between the two walls; $l$ is the length of side wall.
Paper honeycomb is a composite material made of cellular reinforced material and cover paper. It appears as a viscoelastic property. So its modulus of elasticity and shear modulus can be known. It is hypothesized that the load of two neighbor edges per unit length is $F_{1}$ and $F_{2}$ respectively. The strain of the two neighbor edges is the same in the axial direction. It meets Eq.(4) as follow:

$$
\frac{F_{1}}{E \delta_{1}}=\frac{F_{2}}{E \delta_{2}}
$$

where, $\delta_{1}, \delta_{2}$ are the thickness of two edges, $\mathrm{E}$ is the paper's modulus of elasticity.

Bonding force $F_{1}$ and $F_{2}$ is equivalent to bonding force $\sigma_{Z}$ in the axial direction, namely,

$\sigma_{z} \frac{3}{2} l \frac{\sqrt{3}}{2} l=F_{1} l+F_{2} \frac{1}{2} l$

Strain energy of paper can be gotten as follows:

$$
\begin{aligned}
& U_{c}=\int_{0}^{\frac{l}{2}}\left(\frac{F_{1}}{E \delta_{1}}=\frac{F_{2}}{E \delta_{2}}\right) d s \Delta z=\frac{27 l^{3}}{16 E\left(2 \delta_{1}+\delta_{2}\right)} \Delta z \delta_{z}^{2} \\
& =\frac{1}{2} \frac{\sigma_{z}^{2}}{E_{c}} \Delta v
\end{aligned}
$$

Transverse modulus of elasticity can be gotten as follows:

$$
E_{c}=\frac{2}{3 \sqrt{3}}\left(\frac{2 \delta_{1}+\delta_{2}}{l}\right) E
$$

Shear modulus meets Eq.(8) as follows:

$$
G_{x}=\frac{\sqrt{3} \delta G}{2 l}, G_{y}=\frac{\delta G}{\sqrt{3} l}
$$

where, $G$ is the shear modulus of paper, $\delta$ is the thickness of paper honeycomb core.

A paper honeycomb core is made of six edges. Among other things, two edges glued other edges while the left are not. The relation between length of glued edge and other edge meets Eq. (9):

$y=15 \sqrt{3} l^{2}$

where, ${ }^{y}$ is the load-carrying aream, ${ }^{l}$ is the size dimension of paper honeycomb core.

Density of paper honeycomb can be defined as follow:

$\rho=\frac{m}{v}=\frac{30 \sqrt{3} P_{1} l^{2}+2400 P_{2} l+m_{g}}{900 \sqrt{3} l^{2}}$

where, $P_{1}$ is the density of cover paper, $P_{2}$ is the density of honeycomb core, $m_{g}$ is the mass of glue water. 


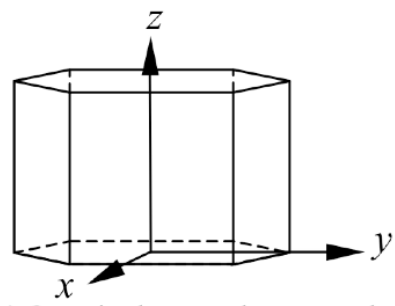

(a) One single paper honeycomb

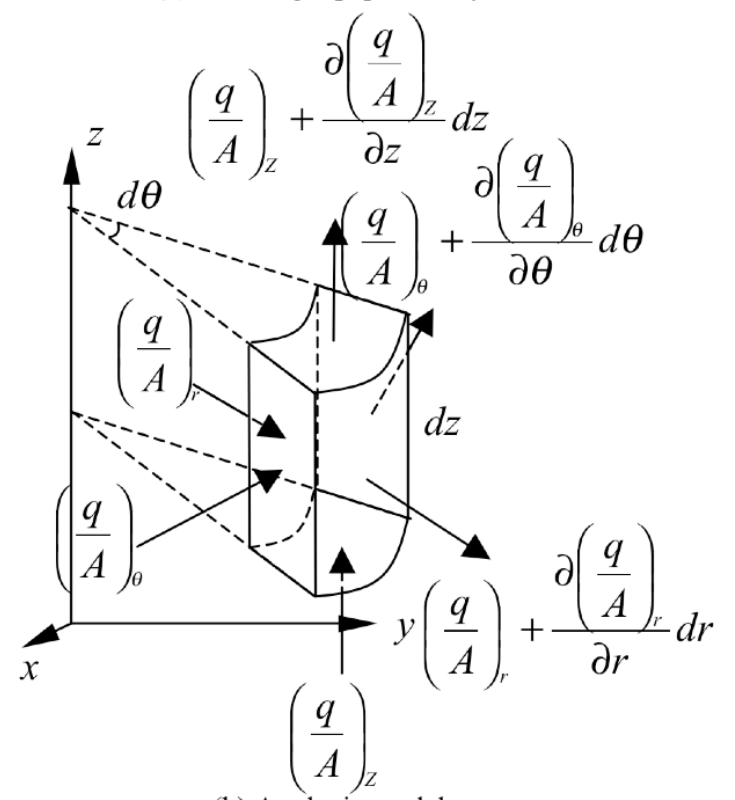

(b) Analysis models

Fig. (2). A heat transfer model of a single honeycomb core.

\subsection{Drying Process of Honeycomb Carton}

When the paper honeycomb core is bending to honeycomb carton, stress concentrations due to bending load and cohesive force caused by glue water should be considered. A method is used for solving this question, namely, after the paper honeycomb cores are applied glue water, bending load is loaded to develop honeycomb carton, then glue water is applied again in bending corner. At last, drying process of honeycomb carton is carried out to fall into a pattern.

For researching of material drying feature, besides the external drying condition, it is more important to study the internal heat and mass transfer mechanism. The drying process of honeycomb carton is actually porous medium drying. Normal solid is considered when the porous substance porosity is zero and normal fluid is considered when the porosity is one, porous substance is considered when the porosity is between zero to one. So the main drying object is moist porous medium $[14,15]$.

To quantitatively describe paper honeycomb internal temperature and moist content distribution and theoretically reveal the heat and mass transfer mechanism of honeycomb carton during drying process, diffusion theory and Fcik diffusion law have been gotten. For establishing the heat and mass transfer model, it has been assumed as follows: (1) The internal temperature and moisture of paper honeycomb distribute uniformly at the beginning of drying; (2) The moisture movement caused by presssure gradient is not taken into consideration; (3) Ignoring paper honeycomb's shrinkage during drying process.

Taking one single paper honeycomb core from paper honeycomb, the hexagon cross section is equivalent to round in favor of theoretical analysis (shown in Fig. 2).

First law of thermodynamics expresses the natural law of energy conservation, namely, energy can be transformed from one form to another form and from one object to another object. But the total energy keeps the same. For any energy transforming system, the energy conservation law can be expressed as follow:

$\rho \frac{d U}{d t} r d r d \theta d z=\rho \frac{d Q_{\text {pure }}}{d t} r d r d \theta d z$
$+\rho \frac{d Q_{\text {inner }}}{d t} r d r d \theta d z$

where, $\rho$ is density of paper honeycomb core, $U$ is the internal energy, $Q$ is the heat flow.

(1) The element net input heat flow rate.

The element net input heat flow rate in $r$ direction can be gotten as follow:

$\left(\frac{q}{A}\right)_{r} r d \theta d z-\left[\left(\frac{q}{A}\right)_{r}+\frac{\partial\left(\frac{q}{A}\right)_{r}}{\partial r} d r\right](r+d r) d \theta d z$

$=-\left(\frac{q}{A}\right)_{r} d r d \theta d z-\frac{\partial\left(\frac{q}{A}\right)_{r}}{\partial r} r d r d \theta d z$

The element net input heat flow rate in $\theta$ direction can be gotten as follow:

$\left\{\left(\frac{q}{A}\right)_{\theta}-\left[\left(\frac{q}{A}\right)_{\theta}+\frac{\partial\left(\frac{q}{A}\right)_{\theta}}{\partial \theta} d \theta\right]\right\} d r d \theta$
$=-\frac{\partial\left(\frac{q}{A}\right)_{\theta}}{\partial \theta} d r d \theta d z$

The element net input heat flow rate in $\mathrm{z}$ direction can be gotten as follow:

$$
\begin{aligned}
& \left\{\left(\frac{q}{A}\right)_{z}-\left[\left(\frac{q}{A}\right)_{z}+\frac{\partial\left(\frac{q}{A}\right)_{z}}{\partial z} d z\right]\right\} r d r d \theta \\
& =-\frac{\partial\left(\frac{q}{A}\right)_{z}}{\partial z} r d r d \theta d z
\end{aligned}
$$


So the element gross net input heat flow rate is $-\left(\frac{q}{A}\right)_{r} d r d \theta d z-\left[\frac{\partial\left(\frac{q}{A}\right)_{r}}{\partial r}+\frac{\partial\left(\frac{q}{A}\right)_{\theta}}{\partial \theta}+\frac{\partial\left(\frac{q}{A}\right)_{z}}{\partial z}\right] r d r d \theta d z$. It can be gotten according to Fourier's Law:

$$
\left(\frac{q}{A}\right)_{r}=-k \frac{\partial T}{\partial r},\left(\frac{q}{A}\right)_{\theta}=-k \frac{\partial T}{\partial \theta},\left(\frac{q}{A}\right)_{z}=-k \frac{\partial T}{\partial z}
$$

where, $k$ is heat conduction of paper honeycomb.

$$
\begin{gathered}
\text { So the heat flow rate is } \\
k\left(\frac{\partial T}{\partial r}+r \frac{\partial^{2} T}{\partial r^{2}}+\frac{\partial^{2} T}{\partial \theta^{2}}+r \frac{\partial^{2} T}{\partial z^{2}}\right) d r d \theta d z \text { which is input into the }
\end{gathered}
$$
element by heat conduction.

(2) The heating rate of internal heat source

There is no heat source inside the element, so the heating rate of internal heat source is zero. According to first law of thermodynamics, the growth rate of the internal energy in the element of paper honeycomb can be gotten as follow:

$$
\begin{aligned}
& \rho \frac{d U}{d t} r d r d \theta d z=\rho \frac{\partial H}{\partial t} r d r d \theta d z \\
& =\rho\left(c_{p} \frac{\partial T}{\partial t}-\frac{1}{1+M} \frac{\partial M}{\partial t} Q_{f g}\right) r d r d \theta d z
\end{aligned}
$$

where, $c_{p}$ is the constant pressure specific heat, $H$ is the enthalpy, $\mathrm{M}$ is the moisture content, $Q_{f g}$ is the latent heat of water evaporation.

According to the heat flow rate and Eq. (16), it can be gotten as follow:

$$
\begin{aligned}
& \rho c_{p} \frac{\partial T}{\partial t}=k\left(\frac{\partial T}{\partial r}+r \frac{\partial^{2} T}{\partial r^{2}}+\frac{\partial^{2} T}{\partial \theta^{2}}+r \frac{\partial^{2} T}{\partial z^{2}}\right) \\
& +\frac{1}{1+M} \frac{\partial M}{\partial t} Q_{f g}
\end{aligned}
$$

For axial symmetry of single paper honeycomb core, the above equation can be transformed to:

$\rho c_{p} \frac{\partial T}{\partial t}=k\left(\frac{1}{r} \frac{\partial T}{\partial r}+\frac{\partial^{2} T}{\partial r^{2}}+\frac{\partial^{2} T}{\partial z^{2}}\right)+\frac{1}{1+M} \frac{\partial M}{\partial t} Q_{f g}$

(3) The heat transfer boundary condition

According to Newton law of cooling, the surface heat flow rate $q_{1}$, which is transferred between the single paper honeycomb surface and the hot blast by way of convective transfer, is met as follow.

$q_{1}=h_{T}\left(T_{a}-T\right)$

where, $h_{T}$ is the convective heat transfer coefficient; $T_{a}$ is the hot blast temperature; $T$ is the surface temperature of single paper honeycomb core.
Meanwhile, the heat flow rate $q_{2}$ of moisture evaporation caused by the moisture evaporation on single paper honeycomb core surface is met as follow:

$q_{2}=\frac{\rho V}{A}\left[Q_{f g}+c_{v}\left(T_{a}-T\right)\right] \frac{1}{1+M} \frac{\partial M}{\partial t}$

where, $c_{v}$ is the steam constant pressure specific heat, $\mathrm{A}$ is the surface area of single paper honeycomb core, $\mathrm{V}$ is the volume of single paper honeycomb core.

The heat flow rate transferred from external surface to internal surface by way of heat conduction can be gotten as follow:

$q_{3}=k \frac{\partial T}{\partial n}$

According to heat conservation principle, $q_{1}=q_{2}+q_{3}$, the heat transfer boundary condition of single paper honeycomb core can be gotten as follow:

$-k \frac{\partial T}{\partial n}=h_{T}\left(T_{a}-T\right)+\frac{\rho V}{A}\left[Q_{f g}+c_{v}\left(T_{a}-T\right)\right] \frac{1}{1+M} \frac{\partial M}{\partial t}$

(4) Mass transfer model of the single paper honeycomb core

In the assumed condition of model establishment, there is no moisture diffused into the glue, so the mass transfer only proceeds in the single paper honeycomb core. According to the Fick's second law of diffusion, the mass transfer equation is met as follow:

$\frac{\partial M}{\partial t}=D\left(\frac{1}{r} \frac{\partial M}{\partial r}+\frac{\partial^{2} M}{\partial r^{2}}+\frac{\partial^{2} M}{\partial z^{2}}\right)$

\section{FEA OF PAPER HONEYCOMB}

\subsection{The Built Analytical Models}
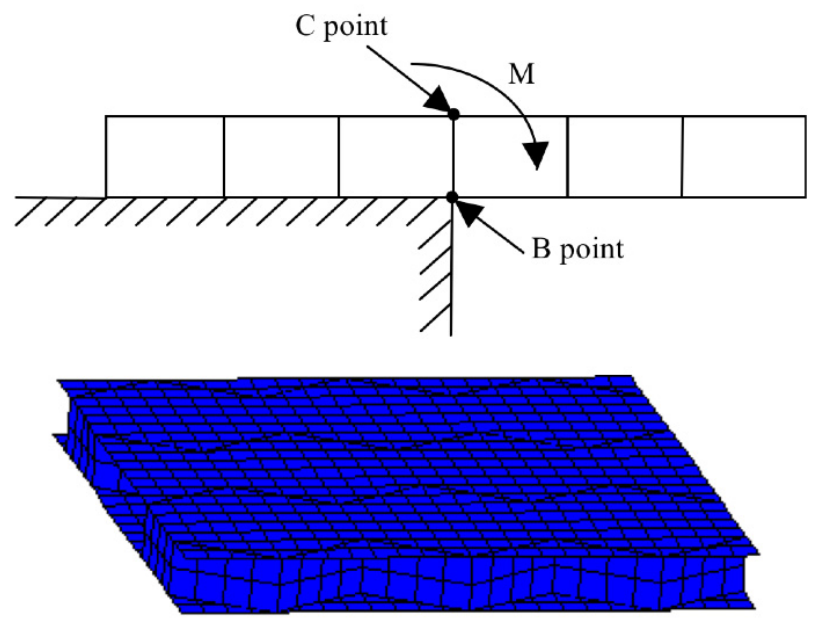

Fig. (3). The analytical model.

The distortion and destroy process of paper honeycomb is very complicated, for it contains material nonlinear or 
structure nonlinear. So its parameters need to be simplified. The simulation of bending course of paper honeycomb is carried out and its stress distribution and deformation course are obtained. The model of paper honeycomb has been built (shown in Fig. 3), which height is $10 \mathrm{~mm}$. The other parameters of the model have been defined in Table 1 .

Table 1. Material parameter of models.

\begin{tabular}{|c|c|c|}
\hline Parameters & Cover Paper & Paper-Core \\
\hline \hline Longitudinal modulus of elasticity & $2.32 \mathrm{GPa}$ & $2.82 \mathrm{GPa}$ \\
\hline Transverse modulus of elasticity & $1.36 \mathrm{GPa}$ & $1.23 \mathrm{GPa}$ \\
\hline Density & $350 \mathrm{~g} / \mathrm{mm}^{2}$ & $230 \mathrm{~g} / \mathrm{mm}^{2}$ \\
\hline Thickness & $0.38 \mathrm{~mm}$ & $0.3 \mathrm{~mm}$ \\
\hline Poisson ration & 0.325 & 0.278 \\
\hline
\end{tabular}

\subsection{Analysis on Bending Process}

The model's classical phases were shown in Fig. (4). The paper-cores only performed bending torque in linear elastic phase. Critical load was reached and it became unsteady and swayed when the load added a little bigger than critical load, which caused buckling. Then steady balance was destroyed. In quick succession, it was compressed sharply near the B point and reached plastic collapse phase. Then the papercores touched each other and entered into densification phase. However, the paper-cores were stretched continuously near the $\mathrm{C}$ point. At last, fracture of cell walls and debonding of honeycomb cores would be happen. The distribution of shear strength had been showed in left of each figure. The value of shear force increased gently before buckling. Then it increased quickly near the bucking point and it increases gently again. At last, it declined quickly after honeycomb cores debonded. The changing curves of maximum shear force and minimum shear force (shown in Fig. 5).

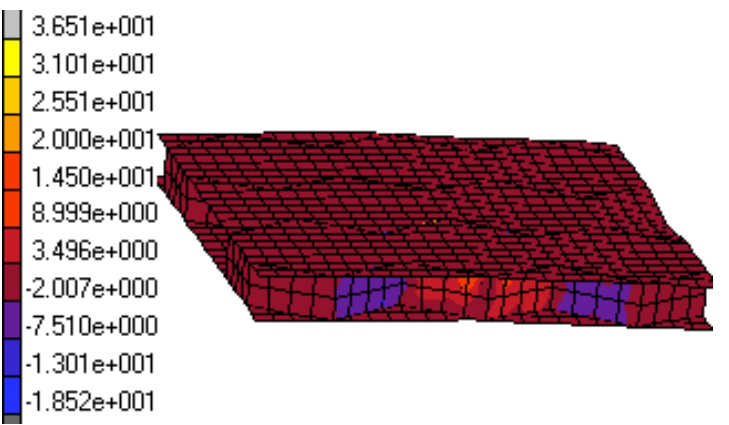

(a) Time is 0.1 second

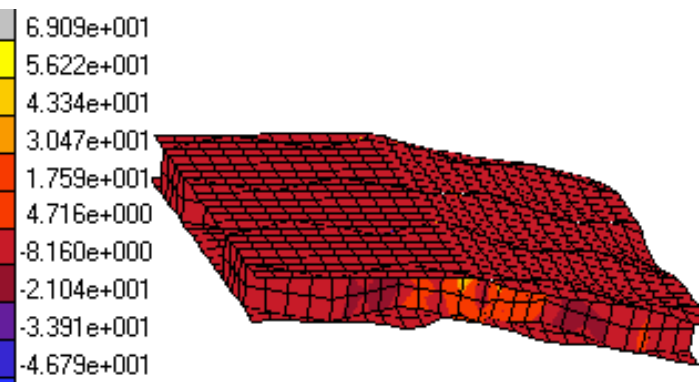

$-5.966 \mathrm{e}+001$

(b) Time is 0.44 second

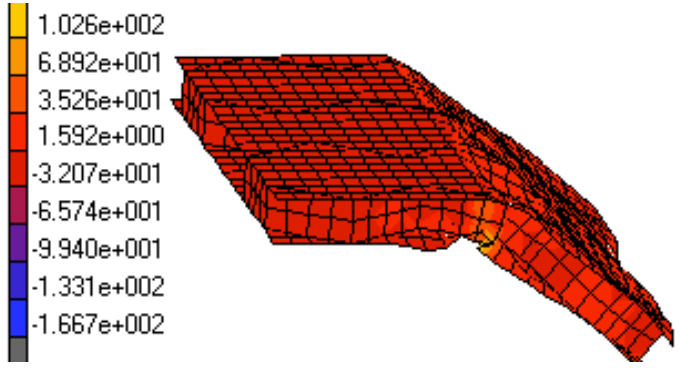

(c) Time is 0.45 second

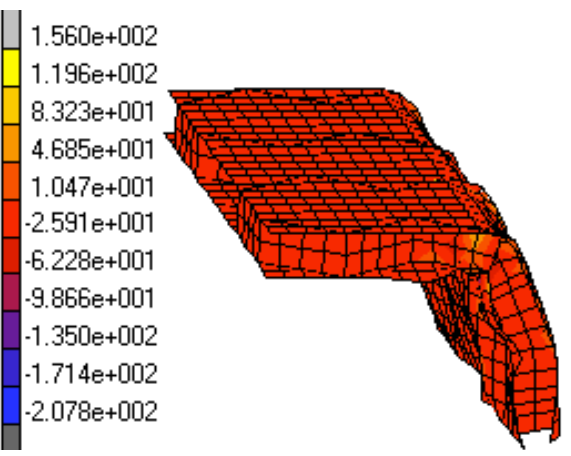

(d) Time is 0.452 second

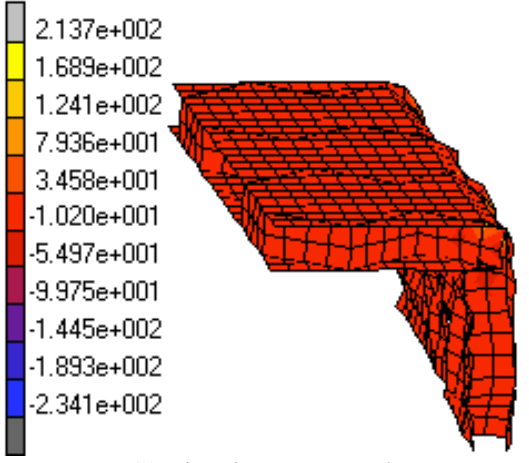

(e) Time is 0.97 second

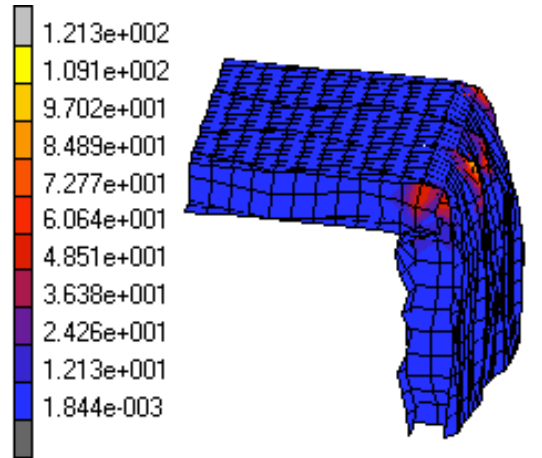

(f) Time is 1 second

Fig. (4). The bending process of paper honeycomb.

The rotation curve in the compression course was showed in Fig. (6). The curve showed that it was linear in linear elastic phase. Angle changed steeply for an instant after bucking. The external load curve of the model was showed in Fig. (7). The curve also showed that it was linear in linear elastic phase. Load changed slowly but displacement changed rapidly in plasticity collapse phase. Displacement almost didn't change along with crescent load in densification phase. It accorded with the ideal displacement-stress curve, which was proved the availability of FEA. 


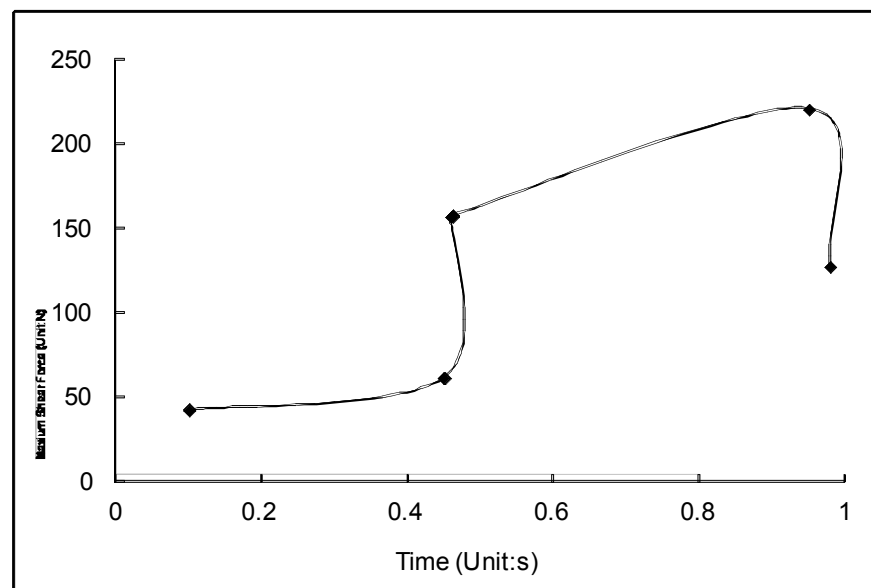

Fig. (5). The changing curve of maximum and minimum shear force.

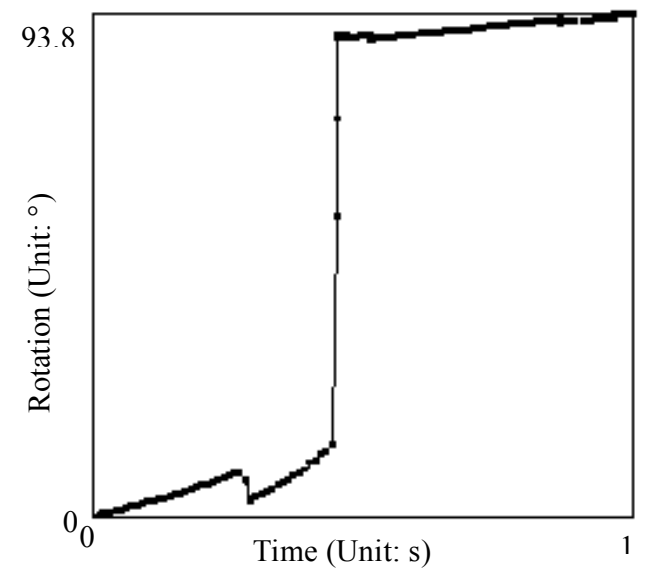

Fig. (6). Rotation curve of the model.

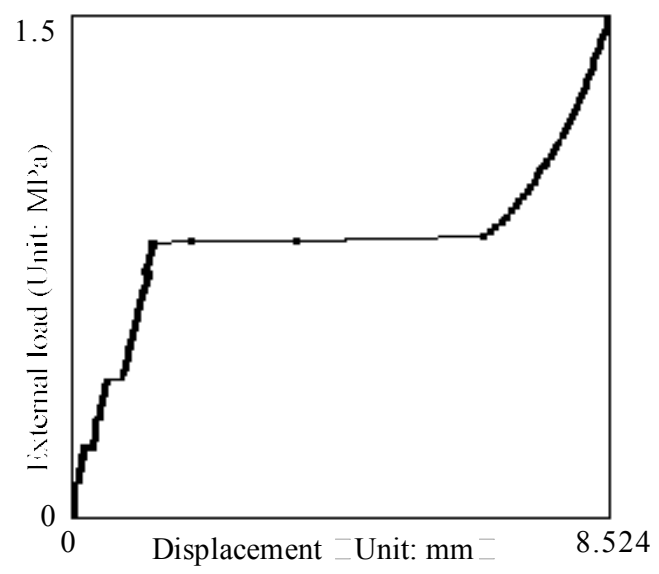

Fig. (7). External load curve of the model.

\section{EXPERIMENTAL VERIFICATION}

The analysis results gotten by FEA had been checked by experimentation. The bending process of paper honeycomb was shown in Fig. (7). The paper-core only performed bending torque in linear elastic phase. It became unsteady and swayed when the load added a little bigger than critical load. In quick succession, the paper honeycomb core near the an-

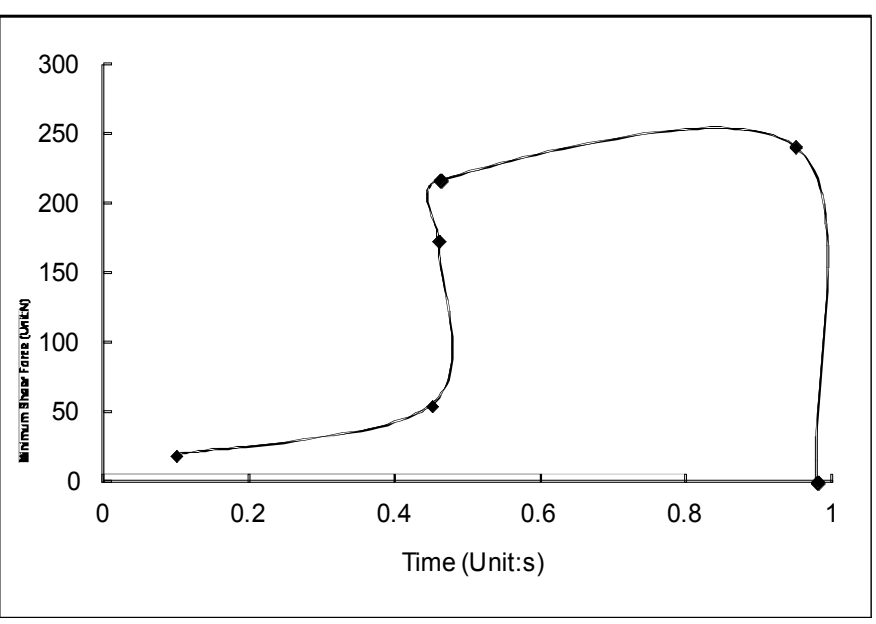

gle table was destroyed and reached plastic collapse phase. Then the paper-core near the angle table touched each other. At last, fracture of paper-core would be happen when the adhesion stress of model was insufficient, even the portion of cover paper would fell away showed in Fig. (8). Stress concentration due to bending load and cohesive force caused by glue water has been happened. So the drying process of honeycomb carton should be carried out to fall into a pattern.

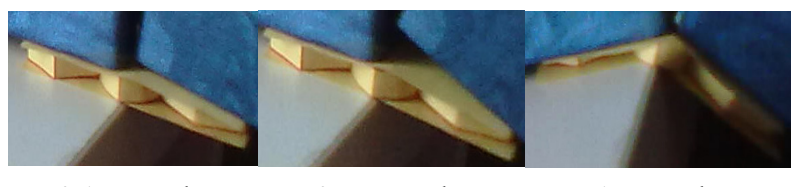
0.1 second
0.5 second
1 second

Fig. (7). The bending course of experimental methods.
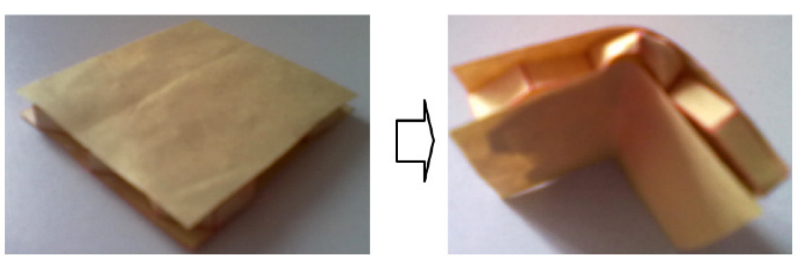

Fig. (8). The bending result of paper honeycomb.

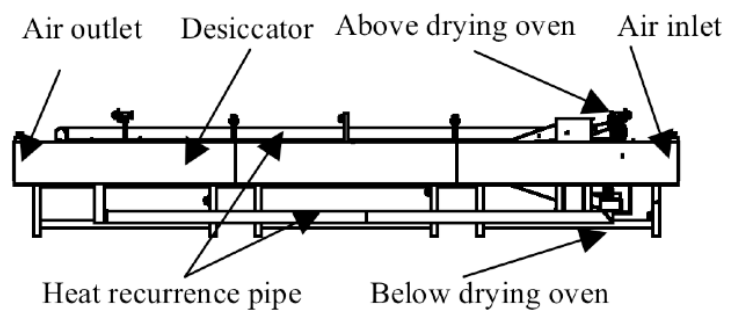

Fig. (9). The structure of drying equipment.

Actual drying equipment has been applied in processing workshop (shown in Fig. 9). Operating principle of drying equipment can be given expression as follow: humid honeycomb cartons bended by a device are put in drying equipment, where two drying ovens offer heat to honeycomb cartons and take water vapor quickly from air inlet to air outlet. Two heat recurrence pipes installed on top and bottom of 
drying equipment, where most heat gets back to drying oven to trap the heat. In addition, partition testing has been made by some devices, such as thermodetector, anemometer and moisture meter.

Drying calculation diagram of honeycomb carton includes enthalpy of moist air, evaporated quantity of water and total heat of drying medium is shown in Fig. (10). Original states of fresh air are follows: environmental temperature $\mathrm{t}_{0}$, moistness $\mathrm{H}_{0}$, heat content $\mathrm{I}_{0}$, total dry air L. Fresh air is heated when it enters air heater (heated state: environmental temperature $\mathrm{t}_{1}$, moistness $\mathrm{H}_{1}$, heat content $\mathrm{I}_{1}$, total dry air $\mathrm{L}$ ). Then moisture percentage of demotes from $\omega 1$ to $\omega_{2}$ and product temperature is up from $t_{\mathrm{m} 1}$ to $t_{\mathrm{m} 2}$ after honeycomb cartons are dried. At last, the air (its state: environmental temperature $\mathrm{t}_{2}$, moistness $\mathrm{H}_{2}$, heat content $\mathrm{I}_{2}$, total dry air L) is back to air heater and steam is exhaust [16].

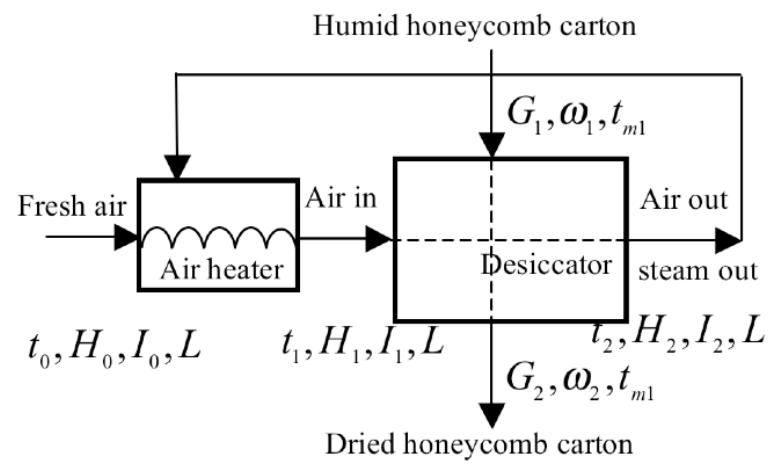

Fig. (10). Drying calculation diagram of honeycomb carton.

It can be gotten the Eq.(24) as follow:

$G_{1}=G_{2} \frac{100-\omega_{2}}{100-\omega_{1}}$

where, $\mathrm{G}_{1}$ is the mass of humid material before entering desiccator, $\mathrm{G}_{2}$ is the mass of material after leaving desiccator. $\omega_{1}$ is moisture percentage of humid material, $\omega_{2}$ is moisture percentage of dried material. low:

Energy balance of drying equipment can be got as fol-

$\Sigma Q+\Sigma W=\Sigma H_{1}-\Sigma H_{2}$

where, $\Sigma Q$ is pure heat flux which enter desiccator, $\Sigma W$ is pure power, $\Sigma H_{1}$ is total enthalpy of feeding product, $\Sigma H_{2}$ is total enthalpy of discharging product.

The statistics has been given in Table 2, which indicated better results by comparison. Now a honeycomb carton had been done by this way shown in Fig. (11).

\section{CONCLUSION}

1. Theoretical analysis on mechanical properties and drying process of paper honeycomb has been gotten, which is integrant to understand the process of honeycomb carton.

2. The FEA on bending course of paper honeycomb is carried out and its stress distribution and deformation course
Table 2. The statistics of drying equipment.

\begin{tabular}{|c|c|}
\hline Relative Humidity (Unit:\%) & $\mathbf{5 9}$ \\
\hline \hline Environment temperature (unit:degree celsius) & 15.7 \\
\hline Initial temperature of paper honeycomb (unit:degree celsius) & 19 \\
\hline Initial humidity ratio (unit:\%) & 42 \\
\hline Final humidity ratio (unit:\%) & 14 \\
\hline
\end{tabular}
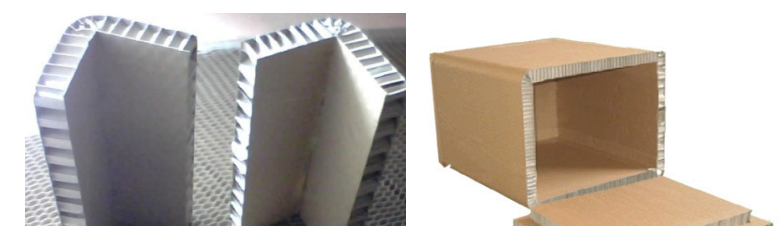

Fig. (11). The product of bended paper honeycombs.

are obtained. The bending process can be divided into four phases: linear elastic phase, elastic-plasticity phase, plasticity collapse phase, densification and fracture phase. A combination of experimental and FEA results have been used to derive a consistent and realistic description of the bending resistance of paper honeycomb.

3. Bending wreckage easily occurs due to stress concentrations which caused by bending load and cohesive force. Drying process of honeycomb carton should be carried out after the bending process.

\section{CONFLICT OF INTEREST}

The authors confirm that this article content has no conflict of interest.

\section{ACKNOWLEDGEMENTS}

Research of this paper is supported by the National Natural Science Foundation of China (Grant No. 41427802) and Zhejiang Industry Polytechnic College.

\section{REFERENCES}

[1] Becker W. "Closed-form analysis of the thickness effect of regular honeycomb core material," Composite Structures, vol.48, pp. 6770, January 2000.

[2] D.H. Chen, S. Ozaki. "Analysis of in-plane elastic modulus for a hexagonal honeycomb core: Effect of core height and proposed analytical method," Composite Structures, vol.88, pp.17-25, March 2009.

[3] Hohe J, BeckerW. "A refined analysis of the effective elasticity tensor for general cellular sandwich cores,". International Journal of Solids and Structures, vol.38, pp.3689-3717, May 2001.

[4] Kobayashi H, Dainaruya M, Kobayashi T. "Dynamic and static compression tests for paper honeycomb cores and absorbed energy," JSME. Series A, Solid Mechanics and Material Engineering, vol.41, pp. 338-344, May 1998.

[5] Wang Dongmei, Wang Zhiwei. "Experimental investigation into the cushioning properties of honeycomb paperboard," Packaging Technology and Science, vol.21, pp.309-316, October 2008.

[6] E Yuping, Wang Zhiwei. "Plateau stress of paper honeycomb as response to various relative humidities," Packaging Technology and Science, vol.23, pp.471-483, December 2010. 
[7] Wang Zhiwei. "Mathematical modeling of energy absorption property for paper honeycomb in various ambient humidities," Materials \& Design, vol.31, pp. 4321-4328, October 2010.

[8] Gibson LJ, Ashby MF. "Cellular solids: structures and properties," Cambridge University Press, 1999.

[9] D.H. Chen. "Bending deformation of honeycomb consisting of regular hexagonal cells," Composite Structures, vol.93, pp.736-746, January 2011

[10] Chung.J,Waas AM. "Compressive response of circular cell polycarborate honeycombs under inplane biaxial static and dynamic is stresses-experiments," International Journal of Impact Engineering, 2002(27):729.

[11] Qiong CHEN. "Research on compressive performance of honeycomb paperboard," Applied Mechanics and Materials, vol.44, pp. 2818-2821, 2011.
[12] Qiong CHEN, Yuan-jun LV. "Drop impact behavior of packaging system with irregular honeycomb core," Advanced Materials Research, vol.146, pp. 127-133, 2011.

[13] Pan SD, Wu LZ, Sun RG, Zhou ZG, Qu JL. "Longitudinal shear strength and failure process of honeycomb cores". Compos Struct, vol.72, pp.42-46, January 2006.

[14] P Chen, P S Schmidt. "An integral model for convective drying of hygroscopic and non-hygroscopic Materials," Drying'89, Washington, D: Hemisphere Pub Corp, pp.162-168, 1990.

[15] MinettM. "A study of air flow effects on the cushioning characteristies of multilayered pre-compressed fiberboard," Doctoral dissertation of Victoria University, 2006

[16] Lv Yuanjun, Chen Lanzhen, Chen Qiong. "Research on drying process of honeycomb paperboard," 2010 International Conference on Measuring Technology and Mechatronics Automation, v 3, pp57-60, 2010.

Received: September 16, 2014

Revised: December 23, 2014

Accepted: December 31, 2014

(C) Chen and Lv; Licensee Bentham Open.

This is an open access article licensed under the terms of the Creative Commons Attribution Non-Commercial License (http://creativecommons.org/licenses/by-nc/3.0/) which permits unrestricted, non-commercial use, distribution and reproduction in any medium, provided the work is properly cited. 